\title{
SPLINTAGE FOR CONGENITAL DISLOCATION OF THE HIP
}

\author{
IS IT SAFE AND RELIABLE? \\ J. BRADLEY, M. WETHERILL, M. K. D. BENSON \\ From the Nuffield Orthopaedic Centre, Headington
}

\begin{abstract}
Two-hundred and fifteen children with 288 unstable hips have been treated by splintage during the first nine months of life. A simple treatment protocol was followed and the aim was to test the safety and reliability of this protocol as well as of the Von Rosen splint and the Pavlik harness. Avascular necrosis occurred in 3.8\% of the splinted hips. Despite splintage, a proportion of children needed operation at a later date.
\end{abstract}

It is widely recognised that early diagnosis of congenital hip instability allows prompt treatment and a high likelihood of a successful outcome. It is also clear that hip splintage may damage the growth plate and the epiphysis. Surprisingly, these risks are imperfectly quantified. Furthermore, a proportion of infants with hip instability fail to develop normal hips despite what appears to be adequate splintage. It is to these problems of safety and reliability that this paper addresses itself.

The diagnosis of neonatal hip instability is essentially clinical, though where doubt exists or risk factors are high, investigation by ultrasound, magnetic resonance imaging or radiography may be helpful. Terms such as "clicking hips" serve to confuse rather than to illuminate.

This paper reports the assessment of those children aged up to nine months who were referred to the Nuffield Orthopaedic Centre with hip instability and for whom splintage was the primary treatment.

\section{PATIENTS AND METHODS}

Most of the patients were seen at, or referred from, the maternity units of the John Radcliffe and Horton General Hospitals at Oxford and Banbury respectively;

J. Bradley, FRCS, Senior Registrar

Addenbrooke's Hospital, Hills Road, Cambridge CB2 2QQ, England.

M. Wetherill, FRCS, Major RAMC, Consultant Orthopaedic Surgeon Queen Elizabeth Military Hospital, Stadium Road, Woolwich, London SE18 4QH, England.

M. K. D. Benson, FRCS, Consultant Orthopaedic Surgeon The Nuffield Orthopaedic Centre, Headington, Oxford OX3 7LD, England.

Requests for reprints should be sent to Mr M. K. D. Benson.

(C) 1987 British Editorial Society of Bone and Joint Surgery $0301-620 \mathrm{X} / 87 / 2052 \$ 2.00$ the remainder were from general practitioners or from other specialists. Patients with chromosomal damage or neuromuscular disorders were excluded from the study. All other children up to the age of nine months whose primary treatment was splintage have been included in the study.

Between January 1978 and December 1983, 215 patients with 288 unstable hips were managed by primary splintage. Of these, 109 affected hips were on the left and 33 on the right; in 73 patients both hips were affected. Follow-up ranged from 2 to 8 years.

Each patient's hips were designated as stable, subluxatable, dislocatable, dislocated but reducible, or dislocated and irreducible. No child with an irreducible hip was treated primarily by splintage. Routine neonatal screening was performed by paediatricians and all suspect hips were referred to the orthopaedic staff who determined whether splintage was necessary. Only one in three of the children so referred was in fact splinted, but each child was followed up to ensure that hip development was clinically and radiologically normal.

The treatment protocol followed was that illustrated in Figure 1. When neonatal examination demonstrated only subluxatability, the child was observed for a week or 10 days to see whether spontaneous stabilisation occurred. If the hip became stable no splintage was used, but the child was reassessed and radiographs taken three months later. If by the time the child was a week or 10 days old the hip was still unstable, the child was treated in a Von Rosen splint. Similarly, all children whose hips were initially dislocated or dislocatable were treated in a Von Rosen splint, provided their hips were fully reducible.

The situation was explained to the parents, and they were given a printed account of the problem, the management and the likely outcome. At one week children in splintage were reviewed and a radiograph 


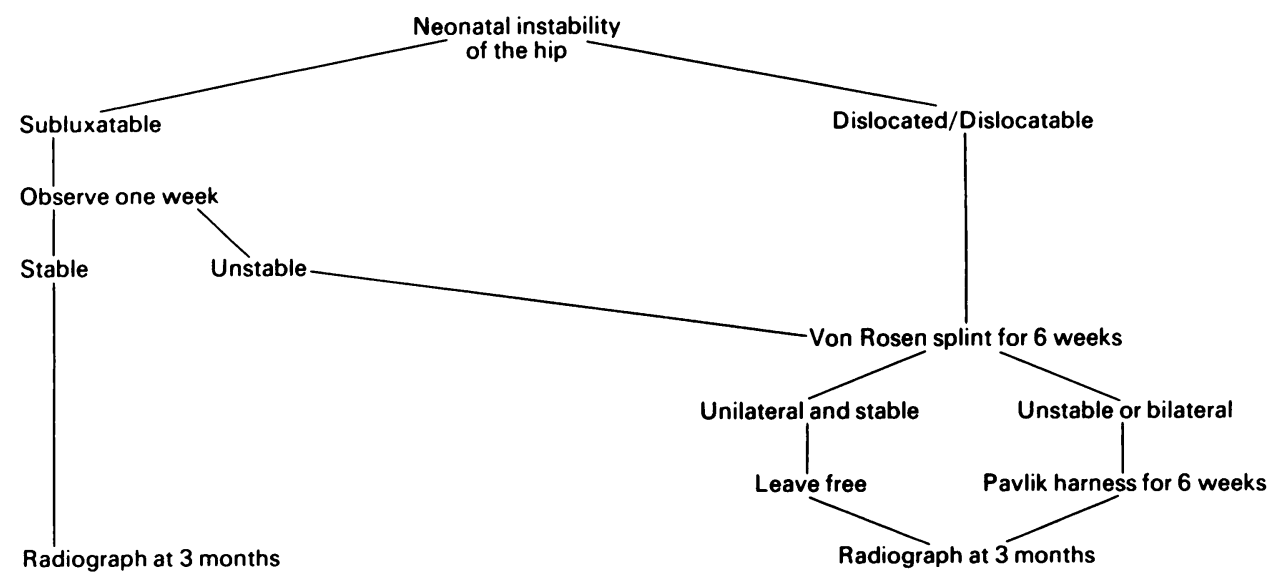

Fig. 1

Flow diagram outlining the treatment of babies with neonatal instability of the hip.

taken to ensure that all was well. The child was checked again three weeks and six weeks after the initial splintage.

At the end of six weeks, the child was examined without the splint. If the dislocation had been unilateral and if hip stability seemed secure, the child was allowed free but encouraged to lie prone with the hips and knees flexed. If at six weeks there was residual instability, or if the dislocation was bilateral, or if there was a family history of instability, a Pavlik harness was applied and worn for a further six weeks; the harness was checked twice during this period. At three months all the children were again assessed clinically and radiologically.

Children who presented with hip instability after the age of six weeks were treated with a Pavlik harness from the start; Von Rosen's splint was not used beyond the age of six weeks for any child. As a rule the Pavlik harness was retained for at least as long as the child's age at presentation; thus, a child presenting at four months would wear a harness for a minimum of four months.

A small group of children who presented late with "dysplastic" hips was assessed arthrographically to ensure that deep reduction was possible; if so, these children were treated in a plaster spica with $100^{\circ}$ of hip flexion, $40^{\circ}$ of abduction and $20^{\circ}$ of medial rotation. The spica was maintained for six weeks and then replaced by a Pavlik harness. Any child who required traction before reduction has not, of course, been included in this study.

Children were assigned to one of three groups depending upon their age at initial treatment. Table I shows the distribution of patients within these three groups as well as the clinical presentation of their hips. The great majority, 171 patients with 239 unstable hips, presented early, and it is clear that most were subluxatable or subluxated. Included in the table, however, are 19 hips which felt normal, had a full range of movement and had no detectable instability; radiologically, however, these hips had poor development of the acetabulum or
Table I. Distribution of hips by clinical presentation and by age at diagnosis

\begin{tabular}{|c|c|c|c|c|}
\hline & $\begin{array}{l}\text { Early } \\
\text { (birth-6 wks) }\end{array}$ & $\begin{array}{l}\text { Intermediate } \\
\text { (7 wks-6 months) }\end{array}$ & $\begin{array}{l}\text { Late } \\
\text { (7-9 months) }\end{array}$ & Total \\
\hline \multicolumn{5}{|l|}{ Subluxatable } \\
\hline Left & 65 & 4 & 4 & 73 \\
\hline Right & 47 & 6 & 2 & 55 \\
\hline Total & 112 & 10 & 6 & 128 \\
\hline \multicolumn{5}{|l|}{ Dislocatable } \\
\hline Left & 34 & 1 & 0 & 35 \\
\hline Right & 14 & 0 & I & 15 \\
\hline Total & 48 & 1 & 1 & 50 \\
\hline \multicolumn{5}{|c|}{ Dislocated but reducible } \\
\hline Left & 51 & 9 & 2 & 62 \\
\hline Right & 28 & 0 & 1 & 29 \\
\hline Total & 79 & 9 & 3 & 91 \\
\hline \multicolumn{5}{|l|}{ Dysplastic } \\
\hline Left & 0 & 10 & 2 & 12 \\
\hline Right & 0 & 6 & $i$ & 7 \\
\hline Total & 0 & 16 & 3 & 19 \\
\hline Total & 239 & 36 & 13 & 288 \\
\hline
\end{tabular}

superolateral subluxation of the femoral head and were thus classified as dysplastic.

The children were again assessed both clinically and radiographically at the age of three months, six months, 12 months and two years. If the progress towards the attainment of a normal hip was then seen to be uneventful, the child was discharged from follow-up. If, however, there were any residual abnormalities such as avascularity or an imperfect acetabulum, they were followed up much longer. In assessing a vascular necrosis, the classifications of Salter, Kostuik and Dallas (1969) and Kalamchi and MacEwen (1980) were used (Table II).

It should be stressed that, whenever splints were applied, great care was exercised to avoid extreme positioning. When a Von Rosen splint was applied, the 


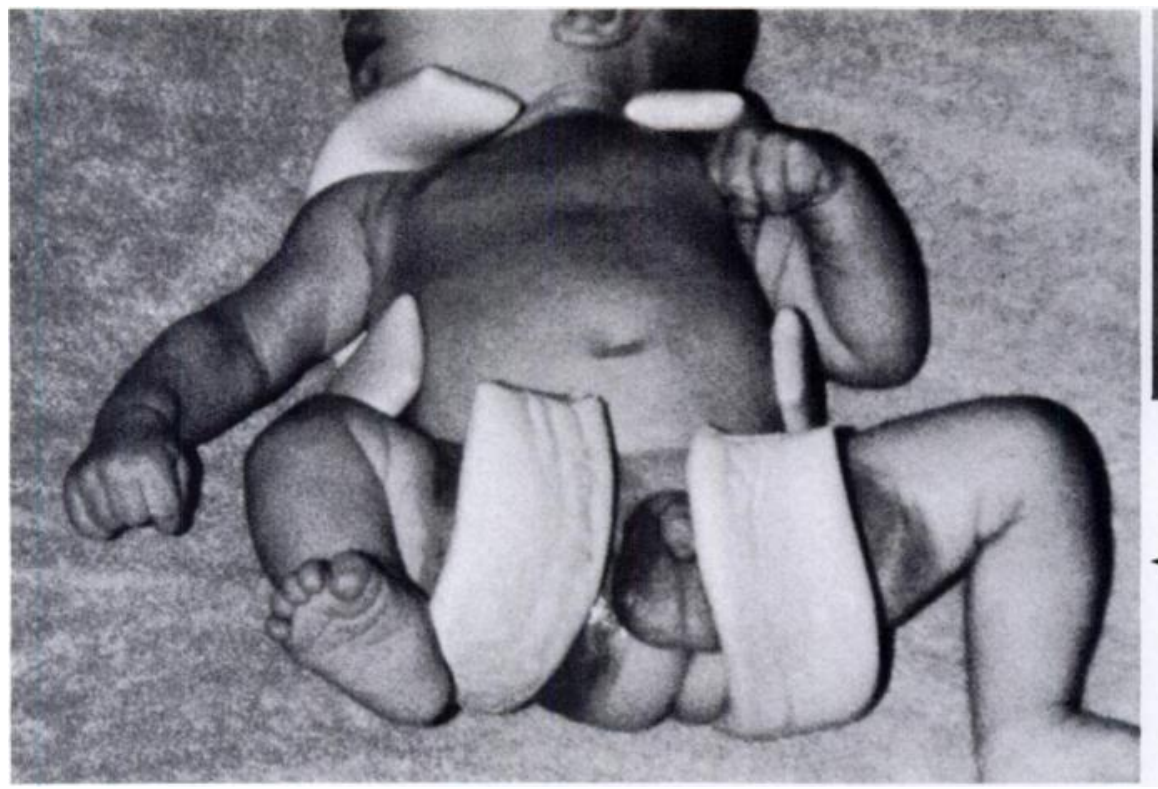

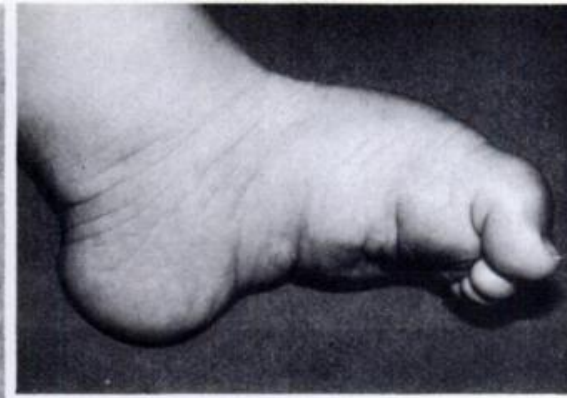

Fig. 3

Fig. 2

Complications of $\mathrm{CDH}$ splintage. Figure $2-$ One baby developed a rash below the sidepieces of a Von Rosen splint; this resolved rapidly when a Pavlik harness was substituted. Figure 3 - Pes cavus after Pavlik harnessing; this took one year to resolve but did so completely. rule was that the child should be able to flex and abduct $30^{\circ}$ beyond the splint and that rotation was free. With the Pavlik harness the hips were flexed between $90^{\circ}$ and $100^{\circ}$ and the posterior strap so arranged as to allow adduction of the hips to neutral when the knee were flexed to $90^{\circ}$. Splintage beyond three months was regarded as prolonged, although older children clearly need longer periods of splintage.

\section{RESULTS}

Close contact between the doctor and the child's family contributed to excellent patient compliance, and in only two patients had the splint been removed or obviously tampered with. Minor pressure sores or rashes were seen in 14 babies using the Von Rosen splint (Fig. 2) and in two using the Pavlik harness. These all responded to minor adjustment or to an earlier transition from Von Rosen to Pavlik splintage. In no instance was the pressure sore anything other than minor and superficial, and no sore failed to heal within two weeks. In babies using the Von Rosen splint the sore was nearly always under the thigh-piece of the splint, and only twice at the shoulder. With the Pavlik harness the sore area in both patients was on the medial side of the knee.

No significant limb swelling occurred after splintage, but several children using the Pavlik harness developed a high arch to the foot (Fig. 3); this deformity, however, did not persist. There were no long-term foot deformities.

We took special note of the failures and complications of splintage. Inability to maintain a stable reduction and to stimulate the normal development of the hip was, of course, regarded as a failure of splintage; so was the necessity for operation. Splintage beyond the expected duration was considered to be a relative failure even though the eventual outcome may have been
Table II. Avascular necrosis: radiological classification

Salter, Kostuik and Dallas 1969

1. Ossific nucleus fails to appear for 1 year.

2. Ossific nucleus fails to enlarge for 1 year.

3. Femoral neck broadening.

4. Ossific nucleus density increases or fragmentation occurs.

5. Long-term deformity of femoral head and/or neck.

Kalamchi and MacEwan 1980

1. Group I: changes (delayed appearances etc.) confined to ossific nucleus.

2. Group II: I plus damage to lateral physis

3. Group III : I plus damage to central physis.

4. Group VI: I plus damage to whole physis.

satisfactory. As for avascular necrosis, this must be regarded as an iatrogenic complication of treatment since spontaneous avascular necrosis in the dislocated hip does not occur (Gage and Winter 1972).

Groups. Early (splintage begun within the first six weeks). Of the 239 hip instabilities (in 171 patients) in this group, eight hips needed operation and 11 required splintage for more than three months. The mean duration of splintage was 10 weeks. Avascular change occurred in three hips.

Intermediate (splintage begun at between six weeks and six months). Of the 36 hips (in 32 children) in this group, six needed operation, 24 required splintage more prolonged than their age at presentation would have suggested, and the mean duration of splintage was 28 weeks. Seven hips developed avascular necrosis.

Late (splintage begun at between seven to nine months). Of the 13 hips (in 12 patients) in this group, four needed operation, eight needed prolonged splintage, and one hip developed avascular necrosis.

These results are summarised in Table III.

The types of operations performed are listed in Table IV. Twenty hips in 17 patients underwent operation; in some children, of course, more than one 
Table III. Results of splintage

\begin{tabular}{|c|c|c|c|c|c|}
\hline & \multicolumn{3}{|l|}{ Group } & \multirow{2}{*}{\multicolumn{2}{|c|}{ Total }} \\
\hline & \multirow{2}{*}{$\begin{array}{l}\text { Early } \\
\text { (birth-6 wks) }\end{array}$} & \multirow{2}{*}{$\begin{array}{l}\text { Intermediate } \\
\text { (7 wks-6 months) }\end{array}$} & \multirow{2}{*}{$\begin{array}{l}\text { Late } \\
\text { (7-9 months) }\end{array}$} & & \\
\hline & & & & Number & Per cent \\
\hline Number of patients & 171 & 32 & 12 & 215 & \\
\hline Number of hips & 239 & 36 & 13 & 288 & \\
\hline $\begin{array}{l}\text { Requiring prolonged splintage } \\
\text { (more than three months) }\end{array}$ & 11 & 24 & 8 & 43 & 14.9 \\
\hline Requiring operation & 10 & 6 & 4 & 20 & 6.9 \\
\hline Avascular necrosis & 3 & 7 & 1 & 11 & 3.5 \\
\hline Mean time of splintage (weeks) & $>10$ & 28 & 23 & & \\
\hline
\end{tabular}

procedure was undertaken. It will be seen that in only one child was open reduction necessary, the majority of procedures being simple tenotomies or femoral osteotomies. In two children, however, a pelvic osteotomy was necessary.

Avascular necrosis occurred in 11 hips $(5 \%$ of the patients or $3.8 \%$ of the unstable hips treated). Table $\mathrm{V}$ shows that in 10 of these 11 patients avascular necrosis was confined to the ossific nucleus and did not affect the growth plate (Figs 4 to 6). In one patient only was the lateral epiphyseal plate affected (Kalamchi Group II): this occurred in a child who presented late (Figs 7 to 9). In five of the 10 hips with Group I changes, the radiological appearance has become completely normal. It is too soon to know if the other five will develop normal hips: two at least seem likely to develop coxa magna.

When the Von Rosen splint was used alone, two of 104 patients developed avascular necrosis $(2 \%)$. When the Von Rosen splint was followed by a Pavlik harness, 1 of 56 patients $(2 \%)$ developed avascular change. When the Pavlik harness alone was utilised as splintage, 5 of 40 $(13 \%)$ showed avascular change.

In a small group of 15 patients the combination of splint and plaster was used, and in them the avascular necrosis rate was $20 \%$; it is difficult to be sure whether the instability or the splintage was responsible, but the immobility imposed by plaster may well have been contributory.

In one baby avascular change developed after a Pemberton osteotomy, and it was unclear whether the initial splintage or the osteotomy or indeed the subsequent plaster was the cause. One baby developed avascular change after Von Rosen splintage in a dislocated position. In one of the 11 cases avascular change developed in the normal hip rather than in the one being treated. No patient developed damage to the central or medial epiphyseal growth plate, so that Kalamchi Group III and Group IV cases were not seen.

In highlighting the problems of splintage it should not be forgotten that there is a high success rate and that those children who present early have a $95 \%$ successful outcome by conservative management alone. It is also
Table IV. Operative treatment of 20 hips in 17 patients

\begin{tabular}{|c|c|c|c|c|}
\hline \multirow[b]{2}{*}{ Operation } & \multicolumn{3}{|l|}{ Group } & \multirow{2}{*}{$\begin{array}{l}\text { Total } \\
\text { operations }\end{array}$} \\
\hline & Early & Intermediate & Late & \\
\hline $\begin{array}{l}\text { Percutaneous adductor } \\
\text { tenotomy }\end{array}$ & 5 & 4 & 3 & 12 \\
\hline $\begin{array}{l}\text { Open adductor } \\
\text { tenotomy }\end{array}$ & 0 & 1 & 0 & 1 \\
\hline $\begin{array}{l}\text { Femoral derotation } \\
\text { osteotomy }\end{array}$ & 8 & 0 & 2 & 10 \\
\hline Open reduction & 1 & 0 & 0 & 1 \\
\hline $\begin{array}{l}\text { Pelvic osteotomy } \\
\text { (1 Pemberton, 1 Salter) }\end{array}$ & 1 & 1 & 0 & 2 \\
\hline Total & 15 & 6 & 5 & 26 \\
\hline
\end{tabular}

reassuring to note that, even in those hips where avascular necrosis developed, the outcome appeared, at least with this short follow-up, to be satisfactory if the changes were confined to the ossific nucleus.

\section{DISCUSSION}

In assessing the reliability of hip splintage the first factor to be considered must be parental compliance and, though parents resent any suggestion that their child is less than perfect, the simple splintage regimen outlined here proved acceptable. A small proportion of children, however, do develop sores with splintage, so careful supervision is obviously essential.

Table V. Avascular necrosis (Kalamchi and MacEwan 1980)

\begin{tabular}{llllc}
\hline & Early & Intermediate & Late & Total \\
\hline Group I & 3 & 7 & 0 & 10 \\
Group II & 0 & 0 & 1 & 1 \\
Group III & 0 & 0 & 0 & 0 \\
Group IV & 0 & 0 & 0 & 0 \\
Total & 3 & 7 & 1 & 11 \\
\hline
\end{tabular}




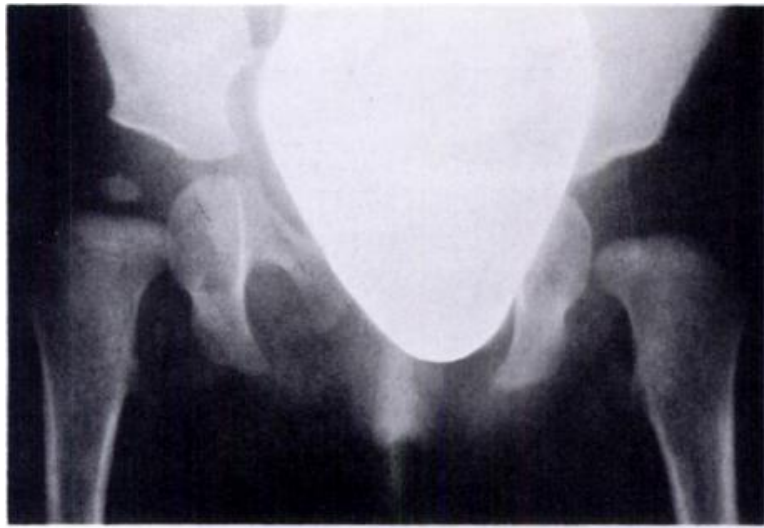

Fig. 4

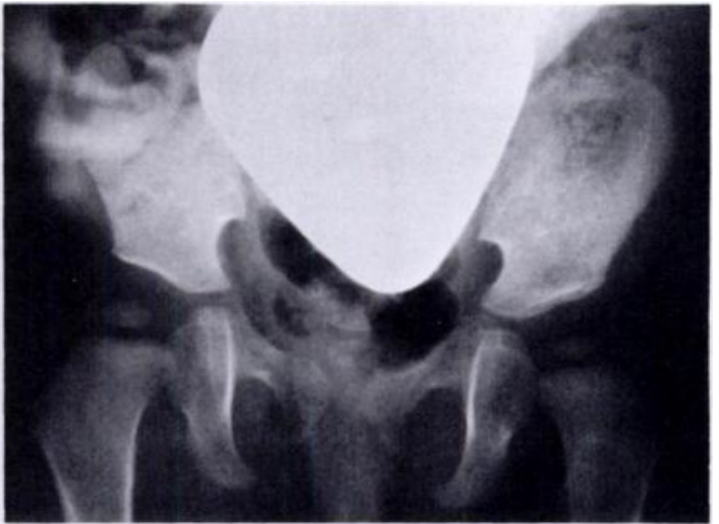

Fig. 5

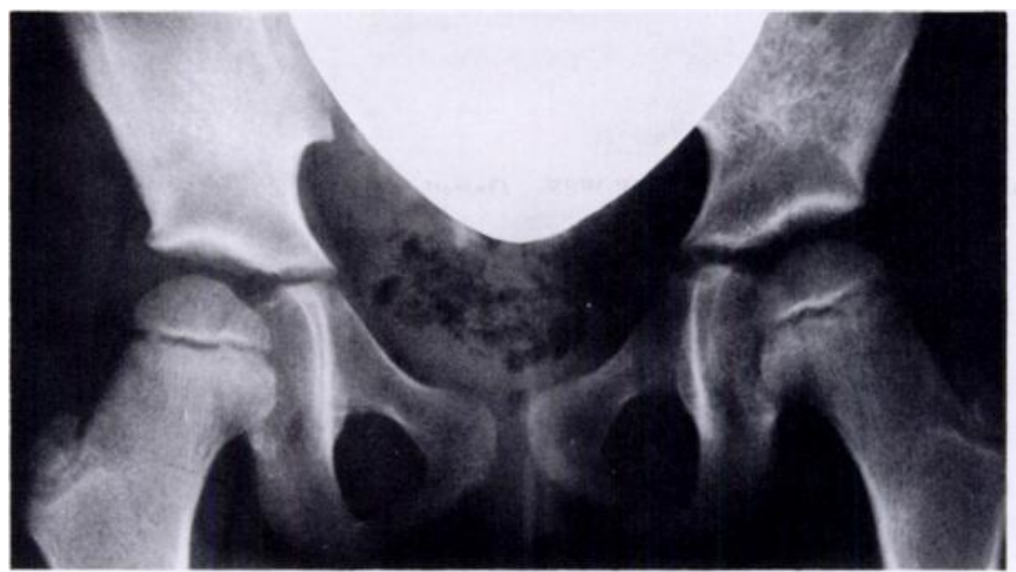

Fig. 6

Figure 4 - In this child treated by a Von Rosen splint, the ossific nucleus of the left hip had not appeared by the age of 13 months. Figure 5 - At 18 months it has appeared but is smaller and more mottled than that of the normal hip. Figure 6 - At five years the epiphysis formed normally but there is slight overgrowth of the femoral neck.

Table VI. Incidence of avascular necrosis

\begin{tabular}{llc}
\hline Authors & Treatment regimen & $\begin{array}{c}\text { Rate of } \\
\text { necrosis } \\
\text { (per cent) }\end{array}$ \\
\hline Salter, Kostuik and Dallas 1969 & $\begin{array}{l}\text { Plaster alone } \\
\text { Plaster after adductor tenotomy }\end{array}$ & $\begin{array}{c}30.0 \\
15.0\end{array}$ \\
Von Rosen 1970 & Von Rosen splint & 1.5 \\
Fredensborg and Nilsson 1976 & Von Rosen splint & 0.18 \\
Westin, Ilfeld and Provost 1976 & $90^{\circ}$ abduction splintage & 15.0 \\
Tönnis 1976 & Pavlik harness & 19.7 \\
Weiner, Hoyt and O'Dell 1977 & Traction, plaster in "human" position & 10.5 \\
Iwasaki 1983 & Pavlik harness as outpatient & 5.8 \\
Elsworth and Walker 1986 & Pavlik harness as inpatient & 28.0 \\
Bradley, Wetherill and Benson 1987 & Denis Browne abduction harness & 9.4 \\
\hline
\end{tabular}




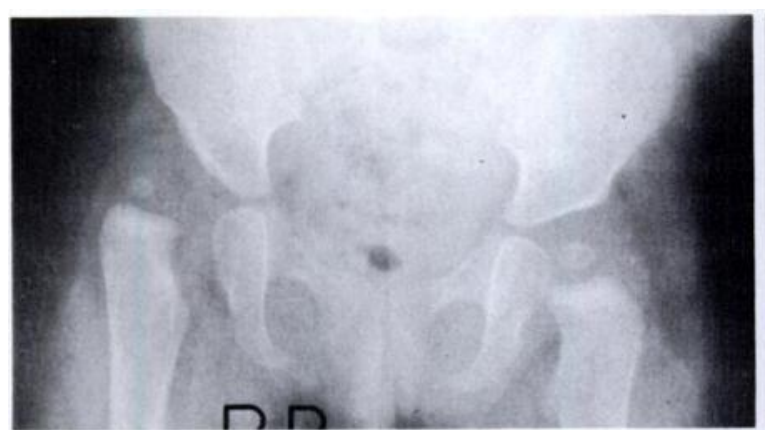

Fig. 7

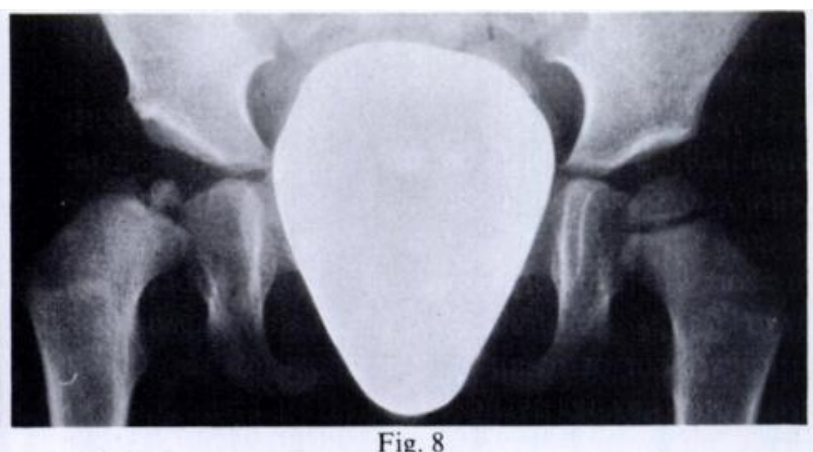

Fig. 8

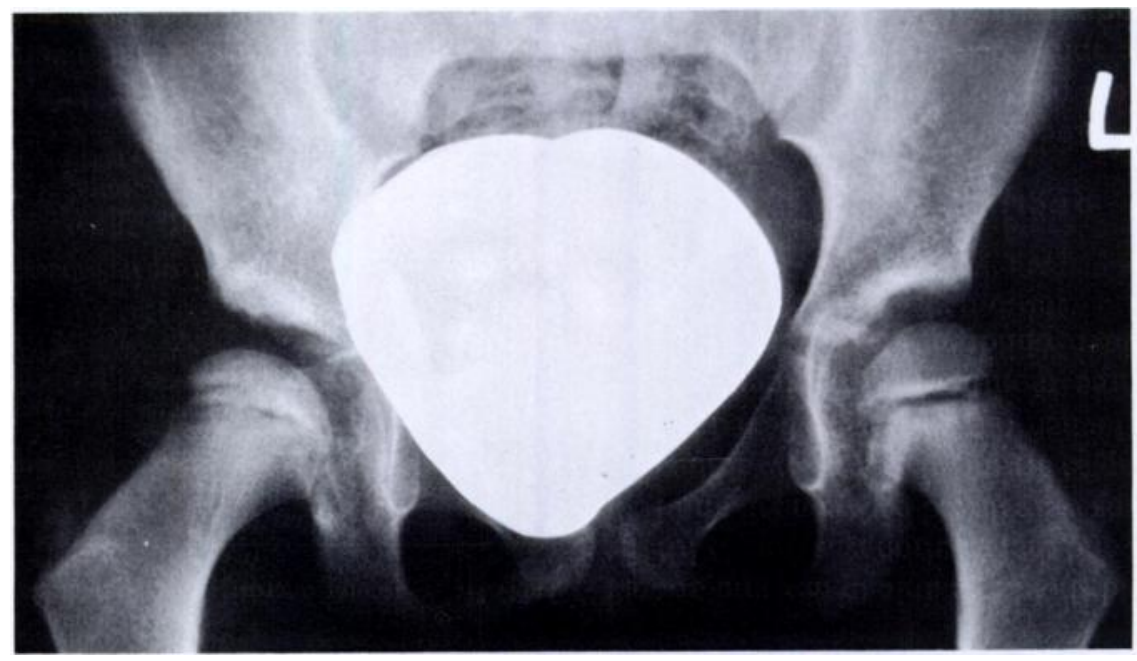

Fig. 9

Figure 7 - This eight-month-old child presented with an established right hip dislocation. Figure 8 - After treatment by a Pavlik harness the right ossific nucleus has failed to mature and there is damage to the lateral physis (Kalamchi, Group II). Figure 9 - By the age of 5 years 6 months the epiphysis is round but has lost height.

It is important to realise that the prompt recognition of $\mathrm{CDH}$ in the neonate, and its treatment by splintage, does not guarantee a normal hip; this has important legal implications. Furthermore, the fact that a group of children who are clinically normal may have radiologically dysplastic hips at three months should be emphasised; 19 children in this series were classified as primarily dysplastic and, without treatment, a proportion would undoubtedly have presented with symptoms later in childhood. The doctor might then be accused of having missed the diagnosis at birth.

Avascular necrosis occurs with every form of hip splintage. Table VI lists some of the recent reports; all were published after the classification of avascular necrosis proposed by Salter et al. (1969). It is clear that forcible manipulation of the hip and its maintenance in any extreme position carries an unacceptably high risk of avascular necrosis. Ogden (1975) described those positions in which the blood supply was most at risk, while Salter et al. (1969) clearly showed that adductor tenotomy offered some protection. In a series of 548 hips treated by Von Rosen splintage, Fredensborg and Nilsson (1976) found only one hip affected by avascular necrosis, but this figure has not been matched by other observers.

Although the Pavlik harness has been used both to achieve reduction and to maintain it, Iwasaki (1983) showed very clearly that strict maintenance of the child in a Pavlik harness in the supine position recreates some of the risks of forced splintage in abduction in plaster: $28 \%$ of his patients treated under strict supervision in hospital developed avascular change in one hip. He concluded that treatment at home with a much more varied posture was safer, and indeed in patients so treated only $5.8 \%$ developed necrosis.

While the medium-term results of avascular necrosis are now established (Kalamchi and MacEwen 1980), there has been no long-term study based on the extent of epiphyseal involvement and the age at which the vascular damage occurred. It is clear, therefore, that there is no room for complacency in the management of the young child with an unstable hip. There is certainly no justification for any form of splintage unless instability is clearly present; the "clicking" hip should not be treated unless instability also is demonstrated. However, even if early diagnosis and treatment cannot guarantee success, most children who are treated by early splintage seem to do very well. 


\section{REFERENCES}

Elsworth C, Walker G. The safety of the Denis Browne abduction harness in congenital dislocation of the hip. J Bone Joint Surg [Br] $1986 ; 68-\mathrm{B}: 275-7$

Fredensborg N, Nilsson BE. Overdiagnosis of congenital dislocation of the hip. Clin Orthop 1976;119:89-92.

Gage JR, Winter RB. Avascular necrosis of the capital femoral epiphysis as a complication of closed reduction of congenital dislocation of the hip: a critical review of twenty years' experience at Gillette Children's Hospital. J Bone Joint Surg [Am] 1972; 54-A :373-88.

Iwasaki K. Treatment of congenital dislocation of the hip by the Pavlik harness: mechanism of reduction and usage. J Bone Joint Surg [Am] 1983;65-A :760-7.

Kalamchi A, MacEwen GD. Avascular necrosis following treatment of congenital dislocation of the hip. J Bone Joint Surg [Am] 1980; 62-A :876-88.

Ogden JA. Treatment positions for congenital dysplasia of the hip. J Pediatr 1975;86:732-4.
Pavlik A. Die funktionelle Behundlungsmethod mittels Riemenbügel als Prinzip der konservativen Therapie bei angeborenen Hüftgelenksverrenkungen der Säuglinge (Method of functional therapy with strap braces as a principle of conservative therapy of congenital dislocation of the hip in infants). Zeitsch Orthop 1957;89:341-52.

Salter RB, Kostuik J, Dallas S. A vascular necrosis of the femoral head as a complication of treatment for congenital dislocation of the hip in young children : a clinical and experimental investigation. Can J Surg 1969;12:44-61.

Tönnis D. An evaluation of conservative and operative methods in the treatment of congenital hip dislocation. Clin Orthop $1976 ; 119: 76-88$.

Weiner DS, Hoyt WA Jr, O'Dell HW. Congenital dislocation of the hip: the relationship of premanipulation traction and age to avascular necrosis of the femoral head. J Bone Joint Surg [Am] 1977;59-A :306-11.

Westin GW, Ilfeld FW, Provost J. Total avascular necrosis of the capital femoral epiphysis in congenital dislocated hips. Clin Orthop 1976;119:93-8.

Von Rosen S. Instability of the hip in the newborn. Act Orthop Scand 1970;Suppl 130:13-24 